\title{
Introduction to the special issue on IEEE NEWCAS 2017
}

\author{
Morgan Madec $^{1}$ (D) $\cdot$ Luc Hebrard $^{1}$ (D) $\cdot$ Wilfried Uhring ${ }^{1}$ (D)
}

Published online: 1 November 2018

(C) Springer Science+Business Media, LLC, part of Springer Nature 2018

This special issue of Analog Integrated Circuits and Signal Processing (ALOG) includes the extended version of a selective number of papers that were originally presented at the past editions of the IEEE International NEWCAS conference, held in Strasbourg (France) on June 25-28, 2017. Since its birth, the IEEE International NEWCAS conference has consistently grown to become a major venue where highly innovative circuit and system design techniques have been presented for the first time. The conference covers a wide variety of subjects including analog and RF circuit design, digital and mixed-signal integrated circuits, computer architecture, signal processing, sensors, encompassing a broad range of applications such as telecommunications, imaging and vision, safety, energy, and biomedical.

In the 2017 NEWCAS edition, the submissions reached the number of 191 from 30 countries from all over the world. Based on a reviewing process with 4 average reviewers per paper, 79 contributions were accepted for presentation with a $47.02 \%$ acceptance rate. This score fits well the general trend of high and yet increasing quality of the conference. This accomplishment is the results of a collective effort supported by the Technical Program Chairs, Dr. Mounir Boukadoum, Dr. Morgan Madec and Pr. Wilfried Uhring, and inspired by the vision of the NEWCAS 2017 General Chairs, Pr. Luc Hébrard and Dr. Mohamad Sawan.

The 11 selected papers included in this special issue address various challenges that are within the scope of NEWCAS, such as Analog/mixed-signal and RF Circuits, Digital and Computing Circuits and Architectures, Digital

Wilfried Uhring

Wilfried.uhring@unistra.fr

Morgan Madec

morgan.madec@unistra.fr

Luc Hebrard

luc.hebrard@unistra.fr

1 ICube, University of Strasbourg and CNRS, 23 rue du Loess, 67037 Strasbourg Cedex, France
Signal Processing and Multimedia, Microsystems, Imaging, and Sensory Circuits and Systems.

We would like to thank the authors for their scientific contributions, and the numerous reviewers for their constructive evaluations. We would also like to express our appreciation for the continuous and tireless support of the Springer personnel. At last, we are grateful to Professor Mohammed Ismail for giving us the opportunity to lead this special issue.

In the end, this special issue was made possible by the personal contribution of many people. We take the opportunity offered by this Editorial to thank each and all of them.

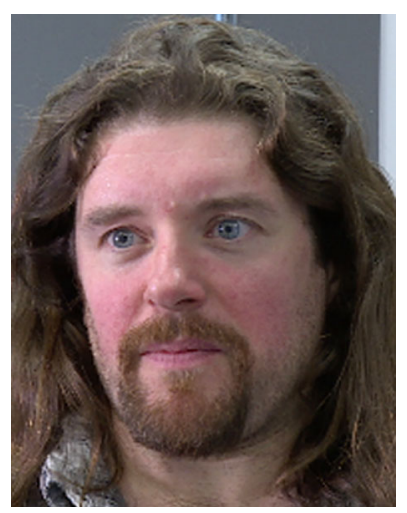

Morgan Madec received the M.Sc. in microelectronics and Ph.D. degrees in mathematics and engineering sciences from the University of Strasbourg, France in 2003 and 2006 respectively. In 2008, he got a position of associate professor at Télécom Physique Strasbourg, an engineering school of the University of Strasbourg. Since, he has been with the "Heterogeneous Systems and Microsystems" team (Electronic department) of the ICube laboratory. $\mathrm{He}$ is the responsible of the "Computer-Aided Design Tools" research activity in this team. His main research fields of interest concerns compact modeling and design tools for multi-domain systems and microsystems, and their extension to new fields such as biotechnologies. During the past 10 years, he was involved in different national and international projects and was in charge of the development of compact models electronic devices (FinFET, CNFET) and integrated sensors (magnetic tunnel junction, Hall-effect sensors, optical sensors, chemical sensors). He also developed a new activity encompassing applications of the interface with biology: biosensors, lab-on-chips, synthetic biology, etc. 


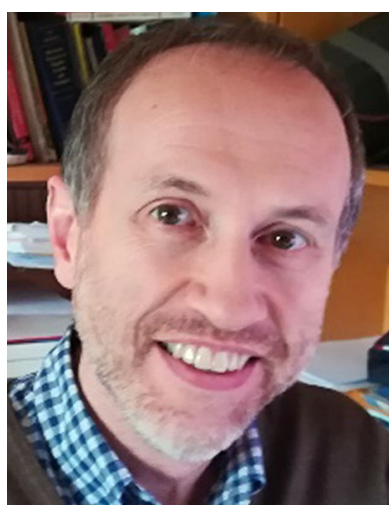

Luc Hebrard is a full Professor at the University of Strasbourg (Unistra) since 2006. He received the Engineer/Master degree and $\mathrm{PhD}$ degree from Ecole Centrale de Lyon in 1990 and 1993 respectively, and the Habilitation for Research Heading (HDR) from University of Strasbourg in 2005. He was a Post-doc at the Centro Nacional de Microelectronica (CNFM Barcelona Spain) from 1994 to 1995, and visiting researcher at Delft University of Technology (Delft - The Netherlands) from 2004 to 2005 . His main research is in the field of integrated sensors (design and modeling) and low noise analog circuit design for instrumentation. He pursues also research in the development of specific CAD tools, especially for electrothermomechanical simulation of integrated circuits. He has coordinated many research projects from the French Agency of Research or in collaboration with industry. L. Hébrard has published more than 100 papers in international journals and conferences. He is member of different international conferences' program committees, and was the General co-Chair of IEEE NEWCAS 2017. He is also the head of the Master in Microelectronics from Unistra.

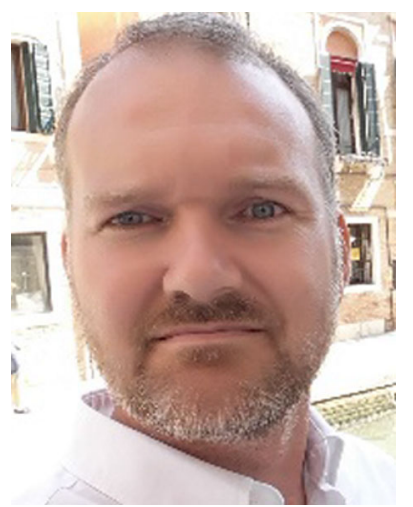

Wilfried Uhring received the M.Sc degree in microelectronic and the Master's Degree in engineering physic in 1999 and the Ph.D. degree in optoelectronics from University of Strasbourg, France, in 2002. Since 1999, he worked on the field of high speed imaging. He designed several ultrafast optical detection devices such as streak camera and gated intensified camera with subnanoseconds to picoseconds resolution. Since 2003, he extend his research activity in integrated ultrafast optoelectronic
CMOS devices such as solid-state streak camera and System on a Chip. In 2013, he joined the Engineering science, computer science and imaging laboratory (ICube) laboratory, university of Strasbourg and CNRS in France as a full professor, where he managed the Heterogeneous System and Microsystem team (SMH) of the ICube laboratory. Wilfried Uhring is member of the Photo-Optical Instrumentation Engineers society (SPIE) and fellow of the IARIA society. $\mathrm{He}$ is involved in the committee of the NEWCAS (IEEE), SENSORCOMM and SIGNAL (IARIA) conferences for several years where he acting as a review tracks leader or in the steering committee. $\mathrm{He}$ is author/coauthor of mare than one hundred peer-reviewed articles in international journals and conferences. 